\title{
Polarization ray picture of coherence for vectorial electromagnetic waves
}

\author{
Alfredo Luis* \\ Departamento de Óptica, Facultad de Ciencias Físicas, Universidad Complutense 28040 Madrid, Spain
}

(Received 10 June 2007; published 18 October 2007)

\begin{abstract}
We elucidate a ray picture of coherence for vectorial electromagnetic waves by using the Stokes parameters for light rays defined by the optical Wigner function. Paraxial propagation is formulated as a complementary Huygens principle. We show that the degrees of coherence are averages of the phase difference where the weights are the Stokes parameters for light rays. We analyze the van Cittert-Zernike theorem for vectorial waves in terms of ray propagation. We show that simple polarization measurements in a Young interferometer determine the degrees of coherence for vectorial electromagnetic waves.
\end{abstract}

DOI: 10.1103/PhysRevA.76.043827

PACS number(s): 42.25.Ja, 42.25.Kb, 42.15.-i, 42.25.Hz

\section{INTRODUCTION}

The proper assessment of coherence for vectorial waves is a very basic and nontrivial issue only recently addressed in depth, so that currently different and partially incompatible approaches coexist [1-5]. Therefore, there are still interesting items to be discovered in this area. In particular, this is the case of the relationship between interferometric coherence and polarization, as the two most relevant optical manifestations of coherence.

In this work we develop the connection between coherence and polarization for vectorial electromagnetic waves. To this end we find especially useful the spatial-angular Stokes parameters defined by the optical Wigner function that represent the polarization state of the rays of geometrical optics [4-10]. In this work we show that this geometrical formulation of the propagation of vectorial light contains coherence properties in a simple and enlightening way. More specifically the contributions of this paper are as follows.

(i) The spatial-angular Stokes parameters provide a simple picture of the propagation of vectorial light which is formally identical to the Huygens principle but in terms of opposite concepts, i.e., rays instead of waves and incoherent superpositions instead of coherent ones (Sec. III). This is a vectorial version of the scalar case examined in Ref. [10].

(ii) Different degrees of coherence for vectorial waves recently introduced in Refs. $[1,2,4,5]$ can be expressed entirely in terms of Stokes parameters (Sec. IV).

(iii) In particular, the degrees of coherence introduced in Refs. [1,2] are averages of the phase difference where the weights are the Stokes parameters of geometrical rays (Sec. IV).

(iv) The Stokes parameters of light rays provide an exceedingly simple picture of the van Cittert-Zernike theorem for vectorial electromagnetic fields (Sec. V). This is the vectorial counterpart of the scalar case examined in Ref. [11].

(v) Simple measurements of the Stokes parameters after a Young interferometer allow us to determine diverse degrees of coherence for vectorial electromagnetic waves (Sec. VI).

\section{STOKES PARAMETERS}

In this section we recall the definitions and mutual relationships of previously introduced generalizations of the

\footnotetext{
*alluis@ fis.ucm.es; http://www.ucm.es/info/gioq
}

Stokes parameters. Throughout we deal with second-order correlation properties of transversal, stationary electric fields fully described in the space-frequency domain by the crossspectral density tensor

$\Gamma_{l, m}\left(\boldsymbol{r}_{1}, \boldsymbol{r}_{2}, \omega\right)=\int d \tau\left\langle E_{l}\left(\boldsymbol{r}_{1}, t+\tau\right) E_{m}^{*}\left(\boldsymbol{r}_{2}, t\right)\right\rangle \exp (i \omega \tau)$,

where $l, m=x, y$, and the angle brackets denote ensemble average. For the sake of simplicity in the following formulas we will not specify the frequency $\omega$. The two-dimensional real vectors $\boldsymbol{r}_{1,2}$ are coordinates in the plane orthogonal to the main propagation direction along axis $z$. We assume that the component of the electric field along axis $z$ can be neglected so that only two transversal field components are necessary. The effectiveness of this approximation for Gaussian fields has been examined in Ref. [12].

The standard Stokes parameters expressing polarization properties at a single spatial point $\boldsymbol{r}$ are the four real quantities

$$
s_{j}(\boldsymbol{r})=\operatorname{tr}\left[\sigma_{j} \boldsymbol{\Gamma}(\boldsymbol{r}, \boldsymbol{r})\right],
$$

for $j=0,1,2,3$, where $\sigma_{j}$ are the Pauli matrices, and $\sigma_{0}$ is the $2 \times 2$ identity matrix. These parameters fully specify the local polarization state at a given spatial point, but provide no information about its evolution.

In order to account both for the actual polarization state and its evolution two sets of generalized Stokes parameters are available. On the one hand, we have the four two-point complex Stokes parameters introduced in Refs. [13]

$$
\tilde{S}_{j}\left(\boldsymbol{r}_{1}, \boldsymbol{r}_{2}\right)=\operatorname{tr}\left[\sigma_{j} \boldsymbol{\Gamma}\left(\boldsymbol{r}_{1}, \boldsymbol{r}_{2}\right)\right]
$$

that include the spatial Stokes parameters as $s(\boldsymbol{r})=\tilde{\boldsymbol{S}}(\boldsymbol{r}, \boldsymbol{r})$, where $s, \tilde{S}$ denote the corresponding four-dimensional vectors.

On the other hand, we have also the four real spatialangular Stokes parameters [5-9]

$$
S_{j}(\boldsymbol{r}, \boldsymbol{p})=\operatorname{tr}\left[\sigma_{j} \boldsymbol{W}(\boldsymbol{r}, \boldsymbol{p})\right],
$$

where $\boldsymbol{W}(\boldsymbol{r}, \boldsymbol{p})$ is the Wigner matrix 
$\boldsymbol{W}(\boldsymbol{r}, \boldsymbol{p})=\left(\frac{k}{2 \pi}\right)^{2} \int d^{2} \boldsymbol{r}^{\prime} \boldsymbol{\Gamma}\left(\boldsymbol{r}-\boldsymbol{r}^{\prime} / 2, \boldsymbol{r}+\boldsymbol{r}^{\prime} / 2\right) \exp \left(i k \boldsymbol{r}^{\prime} \cdot \boldsymbol{p}\right)$,

and $k$ is the wave number in vacuum. The four-dimensional real vector $\boldsymbol{S}(\boldsymbol{r}, \boldsymbol{p})$ contains all optical properties attached to the light ray at point $\boldsymbol{r}$ propagating along the paraxial direction specified by the real vector

$$
\boldsymbol{P}=\left(p_{x}, p_{y}, n\right),
$$

where $n$ is the index of refraction and $\boldsymbol{p}=\left(p_{x}, p_{y}\right)$. The key point of this representation is the extremely simple propagation law of $\boldsymbol{S}(\boldsymbol{r}, \boldsymbol{p})$ in the paraxial domain [7-9]. In particular, under free propagation the spatial-angular Stokes parameters $S^{(z)}(\boldsymbol{r}, \boldsymbol{p})$ at any plane $z$ are related to their counterparts at any other plane $z=0$ in the form [7]

$$
\boldsymbol{S}^{(z)}(\boldsymbol{r}, \boldsymbol{p})=\boldsymbol{S}^{(0)}(\boldsymbol{r}-z \boldsymbol{p} / n, \boldsymbol{p}) .
$$

The $S_{j}(\boldsymbol{r}, \boldsymbol{p})$ parameters are related both with $\boldsymbol{s}(\boldsymbol{r})$ and $\widetilde{S}\left(\boldsymbol{r}_{1}, \boldsymbol{r}_{2}\right)$ in the form

$$
\boldsymbol{s}(\boldsymbol{r})=\int d^{2} \boldsymbol{p} S(\boldsymbol{r}, \boldsymbol{p})
$$

and

$$
\begin{gathered}
\boldsymbol{S}(\boldsymbol{r}, \boldsymbol{p})=\left(\frac{k}{2 \pi}\right)^{2} \int d^{2} \boldsymbol{r}^{\prime} \tilde{\boldsymbol{S}}\left(\boldsymbol{r}-\boldsymbol{r}^{\prime} / 2, \boldsymbol{r}+\boldsymbol{r}^{\prime} / 2\right) \exp \left(i k \boldsymbol{r}^{\prime} \cdot \boldsymbol{p}\right) \\
\tilde{\boldsymbol{S}}\left(\boldsymbol{r}_{1}, \boldsymbol{r}_{2}\right)=\int d^{2} \boldsymbol{p} \boldsymbol{S}(\boldsymbol{R}, \boldsymbol{p}) \exp \left[i k\left(\boldsymbol{r}_{1}-\boldsymbol{r}_{2}\right) \cdot \boldsymbol{p}\right]
\end{gathered}
$$

where

$$
\boldsymbol{R}=\frac{1}{2}\left(\boldsymbol{r}_{1}+\boldsymbol{r}_{2}\right)
$$

\section{COMPLEMENTARY HUYGENS PRINCIPLE FOR VECTORIAL LIGHT}

Relations (2.7) and (2.8) can be regarded as the cornerstone of the ray picture of polarization optics offered by the Wigner-function approach. They can be taken as the basis of a complementary Huygens principle for vectorial light analogous to the standard Huygens principle but with inverted terms (see Ref. [10] for the scalar version). This complementary Huygens principle can be formulated in three steps.

(i) Each point $\boldsymbol{r}$ reached by the light becomes a secondary source of rays propagating along different directions specified by $\boldsymbol{p}$. From the propagation law $(2.7)$ each ray $(\boldsymbol{r}, \boldsymbol{p})$ transports field properties encoded in their spatial-angular Stokes parameters $\boldsymbol{S}(\boldsymbol{r}, \boldsymbol{p})$.

(ii) The disturbance evolves as the result of the incoherent superposition of the rays generated by the secondary sources. This is illustrated by Eq. (2.8) expressing that the intensity and the polarization state at each point $\boldsymbol{r}$ are the result of the simple independent addition of the individual spatial-angular Stokes parameters $S(\boldsymbol{r}, \boldsymbol{p})$ associated to all rays $\boldsymbol{p}$ passing through the same point $\boldsymbol{r}$. We emphasize that the incoherent character of the superposition is a universal key feature of the Wigner formalism, independent of the actual coherence properties of the wave, which are expressed in a different way as shown below.

(iii) For the sake of completeness, this may be completed by the input-output transformation experienced by the spatial-angular Stokes parameters under local inhomogeneous alterations of the phase, intensity, and polarization described by a $4 \times 4$ two-point complex matrix $M\left(\boldsymbol{r}_{1}, \boldsymbol{r}_{2}\right)$

$$
\tilde{\boldsymbol{S}}_{\mathrm{out}}\left(\boldsymbol{r}_{1}, \boldsymbol{r}_{2}\right)=M\left(\boldsymbol{r}_{1}, \boldsymbol{r}_{2}\right) \tilde{\boldsymbol{S}}_{\mathrm{in}}\left(\boldsymbol{r}_{1}, \boldsymbol{r}_{2}\right),
$$

relating the input and output two-point complex Stokes parameters. In the ray picture this becomes [9]

$$
\boldsymbol{S}_{\mathrm{out}}(\boldsymbol{r}, \boldsymbol{p})=\int d^{2} \boldsymbol{p}^{\prime} M_{W}\left(\boldsymbol{r}, \boldsymbol{p}-\boldsymbol{p}^{\prime}\right) \boldsymbol{S}_{\mathrm{in}}\left(\boldsymbol{r}, \boldsymbol{p}^{\prime}\right),
$$

where $M_{W}(\boldsymbol{r}, \boldsymbol{p})$ is the Wigner-Mueller matrix

$$
M_{W}(\boldsymbol{r}, \boldsymbol{p})=\left(\frac{k}{2 \pi}\right)^{2} \int d^{2} \boldsymbol{r}^{\prime} M\left(\boldsymbol{r}-\boldsymbol{r}^{\prime} / 2, \boldsymbol{r}+\boldsymbol{r}^{\prime} / 2\right) \exp \left(i k \boldsymbol{r}^{\prime} \cdot \boldsymbol{p}\right) \text {. }
$$

The price to be paid for the rigorous inclusion of coherent phenomena within this ray picture are the unusual properties of the generalized Stokes parameters for certain rays, i.e., $|\vec{S}|>S_{0}$, where $\vec{S}=\left(S_{1}, S_{2}, S_{3}\right)$, which contrasts with the universally valid relation for the spatial Stokes parameters $s_{0}$ $\geq|\vec{s}|$, where $\vec{s}=\left(s_{1}, s_{2}, s_{3}\right)$ [8]. This occurs for the rays with a Wigner matrix $\boldsymbol{W}(\boldsymbol{r}, \boldsymbol{p})$ that fails to be positive, as the vectorial counterpart of the negativity of Wigner functions in the scalar case. An investigation of the relation between negativity and coherence properties in the scalar case can be found in Refs. [14,15].

\section{DEGREE OF COHERENCE AND STOKES PARAMETERS}

Next we show that recently introduced degrees of coherence for vectorial electromagnetic waves can be completely expressed in terms of the polarization variables recalled in Sec. II. The degree of coherence introduced in Ref. [1] can be expressed as

$$
\mu_{1}^{2}=\frac{\left|\operatorname{tr} \boldsymbol{\Gamma}\left(\boldsymbol{r}_{1}, \boldsymbol{r}_{2}\right)\right|^{2}}{\operatorname{tr} \boldsymbol{\Gamma}\left(\boldsymbol{r}_{1}, \boldsymbol{r}_{1}\right) \operatorname{tr} \boldsymbol{\Gamma}\left(\boldsymbol{r}_{2}, \boldsymbol{r}_{2}\right)}=\frac{\left|\tilde{S}_{0}\left(\boldsymbol{r}_{1}, \boldsymbol{r}_{2}\right)\right|^{2}}{s_{0}\left(\boldsymbol{r}_{1}\right) s_{0}\left(\boldsymbol{r}_{2}\right)} .
$$

Similarly, the degree of coherence introduced in Ref. [2] can be expressed as [16]

$$
\mu_{2}^{2}=\frac{\operatorname{tr}\left[\boldsymbol{\Gamma}\left(\boldsymbol{r}_{1}, \boldsymbol{r}_{2}\right) \boldsymbol{\Gamma}\left(\boldsymbol{r}_{2}, \boldsymbol{r}_{1}\right)\right]}{\operatorname{tr} \boldsymbol{\Gamma}\left(\boldsymbol{r}_{1}, \boldsymbol{r}_{1}\right) \operatorname{tr} \boldsymbol{\Gamma}\left(\boldsymbol{r}_{2}, \boldsymbol{r}_{2}\right)}=\frac{\left|\widetilde{\boldsymbol{S}}\left(\boldsymbol{r}_{1}, \boldsymbol{r}_{2}\right)\right|^{2}}{2 s_{0}\left(\boldsymbol{r}_{1}\right) s_{0}\left(\boldsymbol{r}_{2}\right)} .
$$

A further relation between $\mu_{1,2}$ and polarization variables arises from the fact that Eq. (2.10) allows us to express $\widetilde{\boldsymbol{S}}\left(\boldsymbol{r}_{1}, \boldsymbol{r}_{2}\right)$ as the following average of the phase difference: 


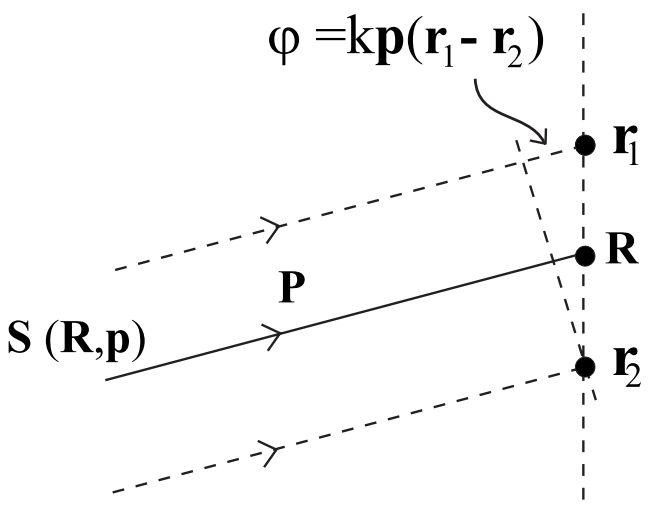

FIG. 1. Picture of the average in Eq. (4.3).

$$
\tilde{\boldsymbol{S}}\left(\boldsymbol{r}_{1}, \boldsymbol{r}_{2}\right)=\int d^{2} \boldsymbol{p} \boldsymbol{S}(\boldsymbol{R}, \boldsymbol{p}) \exp (i \varphi)=\langle\exp (i \varphi)\rangle_{\boldsymbol{S}},
$$

where $\boldsymbol{\varphi}=k \boldsymbol{P} \cdot\left(\boldsymbol{r}_{1}-\boldsymbol{r}_{2}\right)=k \boldsymbol{p} \cdot\left(\boldsymbol{r}_{1}-\boldsymbol{r}_{2}\right)$ is the phase difference $\boldsymbol{\varphi}$ between the points $\boldsymbol{r}_{1}, \boldsymbol{r}_{2}$ produced by a plane wave of wave vector $\boldsymbol{k}=k \boldsymbol{P}$, where $\boldsymbol{P}$ is in Eq. (2.6). The weight of each plane wave $\boldsymbol{p}$ is the spatial-angular Stokes parameters $\boldsymbol{S}(\boldsymbol{R}, \boldsymbol{p})$ for rays at the midpoint $\boldsymbol{R}$ (see Fig. 1).

The square modulus of the first component of Eq. (4.3) provides $\mu_{1}$, while $\mu_{2}$ is given by the square modulus of the complete four-dimensional vector in Eq. (4.3). These are two vectorial analogs of the scalar case examined in Eqs. (36) and (38) of Ref. [10], where it is shown that the degree of coherence for scalar waves is proportional to a phasedifference average where the weight of each plane wave is the corresponding Wigner function at the midpoint. This result is interesting since it agrees well with the physical understanding that partial coherence is related to phase fluctuations, and also agrees with the common interpretation of rays as local plane waves.

Another approach to vectorial coherence [4,5] considers the distance $D$ between the $4 \times 4$ correlation matrix $\boldsymbol{M}$

$$
\boldsymbol{M}=\left(\begin{array}{cc}
\boldsymbol{\Gamma}\left(\boldsymbol{r}_{1}, \boldsymbol{r}_{1}\right), & \boldsymbol{\Gamma}\left(\boldsymbol{r}_{1}, \boldsymbol{r}_{2}\right) \\
\boldsymbol{\Gamma}\left(\boldsymbol{r}_{2}, \boldsymbol{r}_{1}\right) & \boldsymbol{\Gamma}\left(\boldsymbol{r}_{2}, \boldsymbol{r}_{2}\right)
\end{array}\right),
$$

and the $4 \times 4$ identity matrix $I$ representing fully incoherent and unpolarized light

$$
D=\frac{4}{3} \operatorname{tr}\left[\left(\frac{1}{\operatorname{tr} \boldsymbol{M}} \boldsymbol{M}-\frac{1}{4} \boldsymbol{I}\right)^{2}\right]=\frac{4}{3}\left[\frac{\operatorname{tr}\left(\boldsymbol{M}^{2}\right)}{(\operatorname{tr} \boldsymbol{M})^{2}}-\frac{1}{4}\right],
$$

where the numerical factors are introduced for normalization so that $1 \geq D \geq 0$. Then, $D$ can be entirely expressed in terms of the Stokes variables $\boldsymbol{s}(\boldsymbol{r})$ and $\widetilde{\boldsymbol{S}}\left(\boldsymbol{r}_{1}, \boldsymbol{r}_{2}\right)$ since

$$
\begin{gathered}
\operatorname{tr} \boldsymbol{M}=s_{0}\left(\boldsymbol{r}_{1}\right)+s_{0}\left(\boldsymbol{r}_{2}\right), \\
\operatorname{tr}\left(\boldsymbol{M}^{2}\right)=\frac{1}{2}\left[\boldsymbol{s}^{2}\left(\boldsymbol{r}_{1}\right)+\boldsymbol{s}^{2}\left(\boldsymbol{r}_{2}\right)+2\left|\tilde{\boldsymbol{S}}\left(\boldsymbol{r}_{1}, \boldsymbol{r}_{2}\right)\right|^{2}\right] .
\end{gathered}
$$

Finally the overall degree of coherence for vectorial waves $\mu_{G}$ introduced as a weighted average of the local degree of coherence $\mu_{2}$ [17]

$$
\mu_{G}^{2}=\frac{\int d^{2} \boldsymbol{r}_{1} d^{2} \boldsymbol{r}_{2} \operatorname{tr} \boldsymbol{\Gamma}\left(\boldsymbol{r}_{1}, \boldsymbol{r}_{1}\right) \operatorname{tr} \boldsymbol{\Gamma}\left(\boldsymbol{r}_{2}, \boldsymbol{r}_{2}\right) \mu_{2}^{2}\left(\boldsymbol{r}_{1}, \boldsymbol{r}_{2}\right)}{\left[\int d^{2} \boldsymbol{r} \operatorname{tr} \boldsymbol{\Gamma}(\boldsymbol{r}, \boldsymbol{r})\right]^{2}}
$$

can be expressed in terms of polarization variables in the form [18]

$$
\mu_{G}^{2}=\frac{2 \pi^{2}}{k^{2}} \frac{\int d^{2} \boldsymbol{r} d^{2} \boldsymbol{p} \boldsymbol{S}^{2}(\boldsymbol{r}, \boldsymbol{p})}{\left[\int d^{2} \boldsymbol{r} d^{2} \boldsymbol{p} S_{0}(\boldsymbol{r}, \boldsymbol{p})\right]^{2}}
$$

or, equivalently,

$$
\mu_{G}^{2}=\frac{2 \pi^{2}}{k^{2}} \frac{\int d^{2} \boldsymbol{r} d^{2} \boldsymbol{p} S_{0}^{2}(\boldsymbol{r}, \boldsymbol{p})\left[1+\mathcal{P}^{2}(\boldsymbol{r}, \boldsymbol{p})\right]}{\left[\int d^{2} \boldsymbol{r} d^{2} \boldsymbol{p} S_{0}(\boldsymbol{r}, \boldsymbol{p})\right]^{2}},
$$

where

$$
\mathcal{P}^{2}(\boldsymbol{r}, \boldsymbol{p})=\frac{\vec{S}^{2}(\boldsymbol{r}, \boldsymbol{p})}{S_{0}^{2}(\boldsymbol{r}, \boldsymbol{p})} .
$$

It must be noted that $\mathcal{P}$ cannot be straightforwardly interpreted as a degree of polarization because of the properties of $S$ recalled at the end of Sec. III.

\section{VAN CITTERT-ZERNIKE THEOREM}

As a significant example of application of coherence concepts let us address the geometrical picture of the vectorial van Cittert-Zernike theorem expressing the second-order statistical properties of light at a plane $z$ in terms of the same properties at the source plane $z=0$.

Within a purely raylike picture the van Cittert-Zernike theorem for vectorial light is just Eq. (2.7), i.e., $\boldsymbol{S}^{(z)}(\boldsymbol{r}, \boldsymbol{p})$ $=S^{(0)}(\boldsymbol{r}-z \boldsymbol{p} / n, \boldsymbol{p})$, that for fully incoherent sources becomes $\boldsymbol{S}^{(z)}(\boldsymbol{r}, \boldsymbol{p}) \propto \boldsymbol{S}^{(0)}(\boldsymbol{r}-z \boldsymbol{p} / n)$, as a suitable counterpart of the scalar case [11]. This means that the spatial-angular Stokes parameters at point $\boldsymbol{r}$ of the plane $z$ and direction $\boldsymbol{p}$ are the standard spatial Stokes parameters at the point of the source plane connected with $\boldsymbol{r}$ through the ray $\boldsymbol{p}$ (see Fig. 2).

A more wavelike picture can be elaborated from Eq. (2.7) via Eq. (2.10) by expressing in the most general case the two-point complex Stokes parameters $\widetilde{\boldsymbol{S}}^{(z)}\left(\boldsymbol{r}_{1}, \boldsymbol{r}_{2}\right)$ at $z$ in terms of the ray parameters $S^{(0)}(\boldsymbol{r}, \boldsymbol{p})$ at the source plane $z=0$

$\tilde{\boldsymbol{S}}^{(z)}\left(\boldsymbol{r}_{1}, \boldsymbol{r}_{2}\right)=\int d^{2} \boldsymbol{p} \boldsymbol{S}^{(0)}(\boldsymbol{R}-z \boldsymbol{p} / n, \boldsymbol{p}) \exp \left[i k\left(\boldsymbol{r}_{1}-\boldsymbol{r}_{2}\right) \cdot \boldsymbol{p}\right]$.

Then for definiteness, let us consider the quasihomogeneous partially coherent light with

$$
\boldsymbol{\Gamma}\left(\boldsymbol{r}_{1}, \boldsymbol{r}_{2}\right)=\left(\frac{2 \pi}{k}\right)^{2} \boldsymbol{\gamma}(\boldsymbol{R}) \frac{\exp \left[-\left(\boldsymbol{r}_{1}-\boldsymbol{r}_{2}\right)^{2} / A_{c}\right]}{\pi A_{c}},
$$

where $\boldsymbol{R}$ is in Eq. (2.11), $\boldsymbol{\gamma}(\boldsymbol{R})$ is the matrix part of the cross spectral density matrix depending exclusively on $\boldsymbol{R}$, and $A_{c}$ 


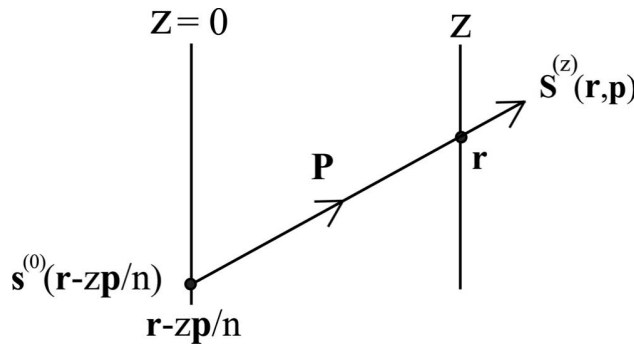

FIG. 2. Illustration of the vectorial version of the van CittertZernike theorem in terms of the spatial-angular Stokes parameters $\boldsymbol{S}^{(z)}(\boldsymbol{R}, \boldsymbol{p})$ at the observation plane $z$ and the spatial Stokes parameters $\boldsymbol{s}^{(0)}(\boldsymbol{r})$ at the source plane $z=0$.

represents the coherence area over the source plane. The case of an incoherent source is obtained in the limit $A_{c} \rightarrow 0$, so that the second factor in Eq. (5.2) tends to a delta function $\delta^{(2)}\left(\boldsymbol{r}_{1}-\boldsymbol{r}_{2}\right)$.

For the cross-spectral density matrix in Eq. (5.2) we get

$$
\boldsymbol{S}^{(0)}(\boldsymbol{r}, \boldsymbol{p})=\boldsymbol{s}_{\gamma}^{(0)}(\boldsymbol{r}) \exp \left(-k^{2} \boldsymbol{p}^{2} A_{c} / 4\right)
$$

where $s_{\gamma}^{(0)}(\boldsymbol{r})$ are the spatial Stokes parameters at the source plane determined by $\boldsymbol{\gamma}$ in the form

$$
s_{\gamma, j}^{(0)}(\boldsymbol{r})=\operatorname{tr}\left[\boldsymbol{\gamma}(\boldsymbol{r}) \sigma_{j}\right]
$$

Finally performing the change of integration variables $\boldsymbol{r}=\boldsymbol{R}$ $-z p / n$ in Eq. (5.1) we get in the limit of an incoherent source $A_{c} \rightarrow 0$

$$
\begin{aligned}
\widetilde{\boldsymbol{S}}^{(z)}\left(\boldsymbol{r}_{1}, \boldsymbol{r}_{2}\right)= & \frac{n^{2}}{z^{2}} \exp \left[i \frac{k n}{2 z}\left(\boldsymbol{r}_{1}^{2}-\boldsymbol{r}_{2}^{2}\right)\right] \int_{-\infty}^{\infty} d^{2} \boldsymbol{r} \boldsymbol{s}_{\gamma}^{(0)}(\boldsymbol{r}) \\
& \times \exp \left[i \frac{k n}{z}\left(\boldsymbol{r}_{2}-\boldsymbol{r}_{1}\right) \cdot \boldsymbol{r}\right],
\end{aligned}
$$

which fully agrees with the wave picture in Ref. [19]. This relation expresses the coherence at plane $z$ as a Fourier transform of the polarization distribution at the source plane.

We can appreciate that Eqs. (5.1) and (5.3) reproduce the structure of coherence as a phase difference average in Eq. (4.3), where the weights are the spatial Stokes parameters $\boldsymbol{s}_{\gamma}^{(0)}(\boldsymbol{r})$, as the whole consequence of the incoherence of the source. The connection between polarization and coherence for partially coherent sources of the form (5.2) can be further analyzed by applying the overall degree of coherence in Eq. (4.8) to this case, leading to

$$
\mu_{G}^{2}=\frac{\pi}{4} A_{c}\left[\frac{\int d^{2} \boldsymbol{r} s_{\gamma}^{2}(\boldsymbol{r})}{\left[\int d^{2} \boldsymbol{r} s_{\gamma, 0}(\boldsymbol{r})\right]^{2}},\right.
$$

which can be expressed also as

$$
\mu_{G}^{2}=\frac{\pi}{4} A_{c} \frac{\int d^{2} \boldsymbol{r} s_{\gamma, 0}^{2}(\boldsymbol{r})\left[1+P^{2}(\boldsymbol{r})\right]}{\left[\int d^{2} \boldsymbol{r} s_{\gamma, 0}(\boldsymbol{r})\right]^{2}},
$$

where $P(\boldsymbol{r})$ is the standard local degree of polarization

$$
P^{2}(\boldsymbol{r})=\frac{\vec{s}_{\gamma}^{2}(\boldsymbol{r})}{s_{\gamma, 0}^{2}(\boldsymbol{r})},
$$

and $\vec{s}=\left(s_{1}, s_{2}, s_{3}\right)$.

The weight $s_{\gamma, 0}^{2}(\boldsymbol{r})$ in Eq. (5.7) is the intensity distribution at the source, and provides a measure of the effective area of the source (see Ref. [20], and references therein). More explicitly, if, for example, $P(\boldsymbol{r})=P$ is constant and $\gamma(\boldsymbol{R})$, $s_{\gamma, 0}(\boldsymbol{r}) \quad$ are $\quad$ Gaussian, $\quad \gamma(\boldsymbol{R}) \propto \exp \left[-\left(\boldsymbol{r}_{1}+\boldsymbol{r}_{2}\right)^{2} / A_{s}\right]$, $s_{\gamma, 0}(\boldsymbol{r}) \propto \exp \left(-4 \boldsymbol{r}^{2} / A_{s}\right)\left[\right.$ with $A_{s} \geq A_{c}$ as a necessary condition for the positivity of $\left.\boldsymbol{\Gamma}\left(\boldsymbol{r}_{1}, \boldsymbol{r}_{2}\right)\right]$ we get that the degree of coherence is proportional to the quotient of the coherence area $A_{c}$ to the effective area of the source $A_{s}$ and to the degree of polarization of the source

$$
\mu_{G}^{2}=\frac{A_{c}}{2 A_{s}}\left(1+P^{2}\right) .
$$

\section{MEASUREMENT}

The relations developed in the preceding sections suggest that polarization measurements may be used to determine in practice the degrees of coherence for vectorial electromagnetic waves. To analyze this point let us focus on the Young interferometer. The two apertures are located at coordinates $\boldsymbol{r}_{1}, \boldsymbol{r}_{2}$ in the plane $z=0$. They are described by Gaussian field-amplitude transmission coefficients identical for both components $t(\boldsymbol{r})=\exp \left[-\boldsymbol{r}^{2} /\left(2 b^{2}\right)\right]$ where $b$ represents the width of the aperture. The width $b$ is assumed to be small enough so that the field is approximately constant within each aperture. In such a case the field after the apertures at $z=0$ is $\boldsymbol{E}^{(0)}(\boldsymbol{r}) \simeq t\left(\boldsymbol{r}-\boldsymbol{r}_{1}\right) \boldsymbol{E}\left(\boldsymbol{r}_{1}\right)+t\left(\boldsymbol{r}-\boldsymbol{r}_{2}\right) \boldsymbol{E}\left(\boldsymbol{r}_{2}\right)$ and the interference depends exclusively on the statistics of the illuminating field just at the two points of interest $\boldsymbol{r}_{1,2}$. Strictly speaking, the limit $b \rightarrow 0$ cannot be taken. This is because the Stokes parameters and the amount of light that crosses the apertures are proportional to the area of the apertures (i.e., proportional to $b^{2}$ ) so that for $b=0$ no light will reach the interference plane.

More specifically, the spatial-angular Stokes parameters just after the apertures at $z=0$ are [5]

$$
\begin{aligned}
\boldsymbol{S}^{(0)}(\boldsymbol{r}, \boldsymbol{p})= & \frac{k^{2} b^{2}}{\pi} \exp \left(-b^{2} k^{2} \boldsymbol{p}^{2}\right)\left\{\boldsymbol{s}\left(\boldsymbol{r}_{1}\right) \exp \left[-\frac{\left(\boldsymbol{r}-\boldsymbol{r}_{1}\right)^{2}}{b^{2}}\right]\right. \\
& +\boldsymbol{s}\left(\boldsymbol{r}_{2}\right) \exp \left[-\frac{\left(\boldsymbol{r}-\boldsymbol{r}_{2}\right)^{2}}{b^{2}}\right] \\
& \left.+\exp \left[-\frac{(\boldsymbol{r}-\boldsymbol{R})^{2}}{b^{2}}\right] \boldsymbol{S}(\boldsymbol{R}, \boldsymbol{p})\right\},
\end{aligned}
$$

where 


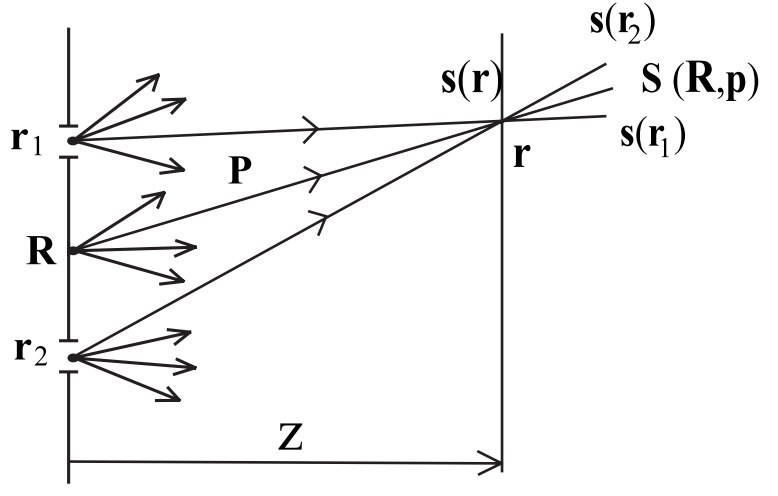

FIG. 3. Illustration of the Young interferometer for vectorial light from the perspective of the Wigner picture leading in the far plane to the relation $\boldsymbol{s}^{(\infty)}(\boldsymbol{r}) \propto \boldsymbol{s}\left(\boldsymbol{r}_{1}\right)+\boldsymbol{s}\left(\boldsymbol{r}_{2}\right)+\boldsymbol{S}(\boldsymbol{R}, \boldsymbol{p}=n \boldsymbol{r} / z)$.

$$
\boldsymbol{S}(\boldsymbol{R}, \boldsymbol{p})=\widetilde{\boldsymbol{S}}\left(\boldsymbol{r}_{1}, \boldsymbol{r}_{2}\right) \exp \left[i k \boldsymbol{p} \cdot\left(\boldsymbol{r}_{2}-\boldsymbol{r}_{1}\right)\right]+\text { c.c. }
$$

and c.c. stands for complex conjugation.

The geometrical picture describes this interference phenomenon as the incoherent superposition of rays originated in three secondary point sources [5]. There are two sources at the apertures with $\boldsymbol{S}^{(0)}\left(\boldsymbol{r}_{j}, \boldsymbol{p}\right) \propto \boldsymbol{s}\left(\boldsymbol{r}_{j}\right), j=1,2$, which are independent of $\boldsymbol{p}$, and a further source at the midpoint between the apertures $\boldsymbol{R}=\left(\boldsymbol{r}_{1}+\boldsymbol{r}_{2}\right) / 2$ with $\boldsymbol{S}^{(0)}(\boldsymbol{R}, \boldsymbol{p}) \propto \boldsymbol{S}(\boldsymbol{R}, \boldsymbol{p})$ (see Fig. 3).

The practical determination of the degrees of coherence via Eqs. (4.1), (4.2), and (4.5)-(4.7) requires the measurement of $\boldsymbol{s}\left(\boldsymbol{r}_{j}\right)$ and $\widetilde{\boldsymbol{S}}\left(\boldsymbol{r}_{1}, \boldsymbol{r}_{2}\right)$. This is the point addressed until the end of the section. The determination of $s\left(\boldsymbol{r}_{j}\right)$ can be achieved by the measurement of the Stokes parameters at the apertures $\boldsymbol{s}^{(0)}\left(\boldsymbol{r}_{j}\right)=\boldsymbol{s}\left(\boldsymbol{r}_{j}\right)$.

On the other hand, the determination of $\boldsymbol{S}(\boldsymbol{R}, \boldsymbol{p})$ and $\widetilde{\boldsymbol{S}}\left(\boldsymbol{r}_{1}, \boldsymbol{r}_{2}\right)$ can be carried out via the measurement of the spatial Stokes parameters $\boldsymbol{s}^{(z)}(\boldsymbol{r})$ at the far field $z \rightarrow \infty$. From Eqs. (2.7) and (2.8) we get

$$
\boldsymbol{S}^{(z)}(\boldsymbol{r})=\int d^{2} \boldsymbol{p} \boldsymbol{S}^{(0)}(\boldsymbol{r}-z \boldsymbol{p} / n, \boldsymbol{p}),
$$

and after a change of variables

$$
\boldsymbol{S}^{(z)}(\boldsymbol{r})=\left(\frac{n}{z}\right)^{2} \int d^{2} \boldsymbol{r}^{\prime} \boldsymbol{S}^{(0)}\left[\boldsymbol{r}^{\prime}, \boldsymbol{p}=n\left(\boldsymbol{r}-\boldsymbol{r}^{\prime}\right) / z\right] .
$$

When $z \rightarrow \infty$ we have that $\boldsymbol{p}=n\left(\boldsymbol{r}-\boldsymbol{r}^{\prime}\right) / z$ tends to be independent of the integration variable $\boldsymbol{r}^{\prime}$ so that $\boldsymbol{p} \rightarrow n \boldsymbol{r} / z$. In such a limit, from Eqs. (6.1) and (6.4) we get

$$
\begin{aligned}
\boldsymbol{s}^{(\infty)}(\boldsymbol{r}) & =\frac{n^{2} k^{2} b^{4}}{z^{2}} \exp \left(-b^{2} k^{2} n^{2} \boldsymbol{r}^{2} / z\right)\left[\boldsymbol{s}\left(\boldsymbol{r}_{1}\right)+\boldsymbol{s}\left(\boldsymbol{r}_{2}\right)+\boldsymbol{S}(\boldsymbol{R}, \boldsymbol{p}\right. \\
& =n \boldsymbol{r} / z)] .
\end{aligned}
$$

This means that after the measurement of the Stokes parameters at the apertures $\boldsymbol{s}\left(\boldsymbol{r}_{1,2}\right)$ and at the far field $\boldsymbol{s}^{(\infty)}(\boldsymbol{r})$ the spatial-angular Stokes parameters $\boldsymbol{S}(\boldsymbol{R}, \boldsymbol{p})$ can be determined for small enough $b$ in the form

$$
\boldsymbol{S}(\boldsymbol{R}, \boldsymbol{p}) \simeq \frac{z^{2}}{n^{2} k^{2} b^{4}}\left[\boldsymbol{s}^{(\infty)}(\boldsymbol{r}=z \boldsymbol{p} / n)-\boldsymbol{s}\left(\boldsymbol{r}_{1}\right)-\boldsymbol{s}\left(\boldsymbol{r}_{2}\right)\right]
$$

In turn, this allows us to determine $\widetilde{\boldsymbol{S}}\left(\boldsymbol{r}_{1}, \boldsymbol{r}_{2}\right)$ via Eq. (6.2). More specifically, since $\widetilde{\boldsymbol{S}}\left(\boldsymbol{r}_{1}, \boldsymbol{r}_{2}\right)$ are eight real quantities it is only necessary to measure the four real point Stokes parameters $\boldsymbol{s}^{(\infty)}(\boldsymbol{r})$ at two properly chosen points in the far plane. For example these can be the points $\boldsymbol{r}_{0, \pi}=z \boldsymbol{p}_{0, \pi} / n$ defined by the conditions

$$
k \boldsymbol{p}_{l} \cdot\left(\boldsymbol{r}_{2}-\boldsymbol{r}_{1}\right)=\frac{n k}{z} \boldsymbol{r}_{l} \cdot\left(\boldsymbol{r}_{2}-\boldsymbol{r}_{1}\right)=\frac{l}{2},
$$

for $l=0, \pi$. In such a case from Eq. (6.2) it follows that

$$
\begin{gathered}
\boldsymbol{S}\left(\boldsymbol{R}, \boldsymbol{p}_{0}\right)=\tilde{\boldsymbol{S}}\left(\boldsymbol{r}_{1}, \boldsymbol{r}_{2}\right)+\widetilde{\boldsymbol{S}}^{*}\left(\boldsymbol{r}_{1}, \boldsymbol{r}_{2}\right), \\
\boldsymbol{S}\left(\boldsymbol{R}, \boldsymbol{p}_{\pi}\right)=i\left[\tilde{\boldsymbol{S}}\left(\boldsymbol{r}_{1}, \boldsymbol{r}_{2}\right)-\widetilde{\boldsymbol{S}}^{*}\left(\boldsymbol{r}_{1}, \boldsymbol{r}_{2}\right)\right]
\end{gathered}
$$

and then

$$
\widetilde{\boldsymbol{S}}\left(\boldsymbol{r}_{1}, \boldsymbol{r}_{2}\right)=\frac{1}{2}\left[\boldsymbol{S}\left(\boldsymbol{R}, \boldsymbol{p}_{0}\right)-i \boldsymbol{S}\left(\boldsymbol{R}, \boldsymbol{p}_{\pi}\right)\right] .
$$

This is equivalent to the polarization resolved visibility considered for $\mu_{2}$ in Ref. [16], but focusing on the ray picture.

In this regard let us also notice that from Eq. (6.2)

$$
S_{j, \max }(\boldsymbol{R}, \boldsymbol{p})=2\left|\tilde{S}_{j}\left(\boldsymbol{r}_{1}, \boldsymbol{r}_{2}\right)\right|,
$$

for $j=0,1,2,3$, where the maximum refers to variations of $\boldsymbol{p}$, and it must be taken into account that the maximum for each $j$ may be obtained for different values of $\boldsymbol{p}$. Thus, alternative expressions for the degrees of coherence are

$$
\mu_{1}^{2}=\frac{\left|S_{0, \max }(\boldsymbol{R}, \boldsymbol{p})\right|^{2}}{4 s_{0}\left(\boldsymbol{r}_{1}\right) s_{0}\left(\boldsymbol{r}_{2}\right)}
$$

and

$$
\mu_{2}^{2}=\frac{\left|\boldsymbol{S}_{\max }(\boldsymbol{R}, \boldsymbol{p})\right|^{2}}{8 s_{0}\left(\boldsymbol{r}_{1}\right) s_{0}\left(\boldsymbol{r}_{2}\right)} .
$$

\section{CONCLUSIONS}

We have elaborated a basic connection between coherence and polarization for vectorial electromagnetic waves. This connection is expressed in terms of the spatial-angular polarization variables defined from the optical Wigner function and attached to the geometrical rays. This analysis benefits from the simple propagation law satisfied by the spatialangular Stokes parameters.

This allows a simple formulation of vectorial light propagation entirely in terms of the incoherent superposition of generalized rays, expressed by a suitable analog of the Huygens principle. We have shown that some of the recently introduced definitions of the degree of coherence are averages of the phase difference, where the weights are the 
spatial-angular Stokes parameters. This formulation provides also a simple picture of the van Cittert-Zernike theorem for vectorial waves in terms of the polarization state at the source. Moreover, from a practical perspective we have shown that the measurement of the polarization state at the apertures and at the far field of a Young interferometer provides the degrees of coherence for the input beam illuminating the apertures.

\section{ACKNOWLEDGMENT}

A. Luis acknowledges the support from Project No. PR1-A/07-15378 of the Universidad Complutense.
[1] B. Karczewski, Phys. Lett. 5, 191 (1963); E. Wolf, Phys. Lett. A 312, 263 (2003); S. A. Ponomarenko and E. Wolf, Opt. Commun. 227, 73 (2003); E. Wolf, Opt. Lett. 29, 1712 (2004).

[2] J. Tervo, T. Setälä, and A. T. Friberg, Opt. Express 11, 1137 (2003); T. Setälä, J. Tervo, and A. T. Friberg, Opt. Lett. 29, 328 (2004); 29, 1713 (2004).

[3] H. M. Ozaktas, S. Yüksel, and M. A. Kutay, J. Opt. Soc. Am. A 19, 1563 (2002); P. Réfrégier and F. Goudail, Opt. Express 13, 6051 (2005); P. Réfrégier and J. Morio, J. Opt. Soc. Am. A 23, 3036 (2006).

[4] A. Luis, J. Opt. Soc. Am. A 24, 1063 (2007).

[5] A. Luis, J. Opt. Soc. Am. A 23, 2855 (2006).

[6] E. C. G. Sudarshan, Physica A 96, 315 (1979); Phys. Rev. A 23, 2802 (1981).

[7] A. Luis, Opt. Commun. 246, 437 (2005).

[8] A. Luis, Opt. Commun. 251, 243 (2005).

[9] A. Luis, Opt. Commun. 263, 141 (2006).

[10] A. Luis, Eur. J. Phys. 28, 231 (2007).
[11] R. Simon, Pramana 20, 105 (1983); H. M. Pedersen, J. Opt. Soc. Am. A 8, 176 (1991); 8, 1518 (1991).

[12] R. Simon, E. C. G. Sudarshan, and N. Mukunda, Appl. Opt. 26, 1589 (1987).

[13] J. Ellis and A. Dogariu, Opt. Lett. 29, 536 (2004); O. Korotkova and E. Wolf, ibid. 30, 198 (2005); J. Mod. Opt. 52, 2659 (2005).

[14] A. Luis, Opt. Commun. 266, 426 (2006).

[15] A. Luis, Opt. Lett. 31, 3644 (2006).

[16] T. Setälä, J. Tervo, and A. T. Friberg, Opt. Lett. 31, 2208 (2006); 31, 2669 (2006).

[17] J. Tervo, T. Setälä, and A. T. Friberg, J. Opt. Soc. Am. A 21, 2205 (2004).

[18] A. Luis, J. Opt. Soc. Am. A 24, 2070 (2007).

[19] F. Gori, M. Santarsiero, R. Borghi, and G. Piquero, Opt. Lett. 25, 1291 (2000); M. A. Alonso, O. Korotkova, and E. Wolf, J. Mod. Opt. 53, 969 (2006).

[20] A. Luis, Phys. Rev. A 66, 013806 (2002); Opt. Lett. 31, 3644 (2006); Opt. Commun. 273, 173 (2007). 